\title{
Knowledge, skills and attitudes of doctors towards assessing cognition in older patients in the emergency department
}

\author{
Sean P Kennelly, ${ }^{1}$ Deirdre Morley, ${ }^{1}$ Tara Coughlan, ${ }^{1}$ Ronan Collins, ${ }^{1}$ Martin Rochford, ${ }^{2}$ \\ Desmond O'Neill ${ }^{1}$
}

\begin{abstract}
Purpose of the study Although cognitive impairment and delirium are highly prevalent in older patients who present to the emergency department, multiple studies have highlighted inadequate detection by doctors. This study investigated potential reasons underlying this.

Study Design A 14-item self-administered questionnaire was distributed to all medical, surgical and emergency department physicians involved in the care of older patients in the emergency department of an urban university teaching hospital between January and March 2012.

Results The questionnaire was completed by $76 / 97$ (78\%) of eligible respondents. Respondents reported screening an average of one in four older patients that they reviewed. Almost one-third $(22 / 76,29 \%)$ felt they lacked the relevant expertise to perform cognitive screening: those with training in geriatrics were less likely to cite lack of expertise as a factor. While the majority felt screening for cognition in the emergency department-setting was important $(59 / 76,78 \%)$, several limiting factors were identified: lack of a screening tool; lack of privacy; too much noise; and time constraints. There was no consensus on who should perform screening.

Conclusions Doctors reviewing patients in the emergency department-setting reported several important factors limiting their ability to screen older patients for cognitive impairment. Respondents to this questionnaire did not feel the emergency department environment was conducive towards the assessment of cognition in older patients. Clarification of each discipline's responsibility in the detection, assessment and management of delirium and/or dementia, and the implementation of emergency department cognitive screening instruments more suited to this setting would likely improve detection and management.
\end{abstract}

\section{INTRODUCTION}

Older patients represent an increasing population in emergency medicine, accounting for up to $24 \%$ of all Emergency Departments (ED) visits. ${ }^{1}{ }^{2}$ They visit ED more frequently than younger adults, ${ }^{3}$ and are at an increased risk for functional decline, adverse effects of treatment, poorer management of pain and repeat visits. ${ }^{45}$ Upon arrival in the ED, their care is often managed at multiple points of care and by multiple individuals as they progress from initial assessment and triage, to assessment by an ED physician, and in many instances further assessment by medical and surgical specialists.
Healthcare personnel working in the ED setting are less comfortable treating and managing older patients. ${ }^{6}$ This may be of major significance in terms of the management of common and serious syndromes of later life, such as cognitive impairment.

There is a high prevalence of cognitive impairment (including combinations of acute cognitive impairment attributable to delirium; chronic cognitive impairment with functional decline attributable to dementia or mild cognitive impairment with no functional decline) in older ED patients. Patients with pre-existing dementia are at a higher risk of developing delirium when acutely unwell. Many factors, including treatment in noisy, congested corridors after a prolonged wait, may contribute to the exacerbation or onset of delirium, or to the worsening of a pre-existing diminished cognitive reserve. ${ }^{7}$ Up to $42 \%$ of older ED patients have cognitive impairment when formal cognitive testing is performed, ${ }^{8-10}$ and on average $10 \%$ of older ED patients will meet the criteria for delirium. ${ }^{11} 12$

Multiple studies have highlighted inadequate detection and reporting of cognitive impairment in older ED patients, ${ }^{12-14}$ with less than a third of cases identified by doctors. Failure of physicians to identify and highlight cognitive impairment can lead to disastrous consequences, including higher rates of rehospitalisation, prolonged hospitalisation and increased morbidity and mortality. ${ }^{15}$ Early diagnosis and treatment of dementia may result in slower cognitive decline and preserved function. ${ }^{16}{ }^{17}$ Many of these patients may have undiagnosed dementia, and their attendance in the ED represents a vital opportunity to intervene early in the course of their disease. Studies have highlighted the reluctance of ED physicians to alter patient care, even when alerted to the presence of cognitive impairment, and primary care physicians have demonstrated a similar reluctance to diagnose and commence treatment for dementia. ${ }^{14} 18$

Despite the existence of several suitable and validated brief cognitive screening tools, no instrument has been widely adopted. ${ }^{19}$ The objective of this study was to assess the knowledge, skills and attitudes of doctors who care for older patients in the $E D$, regarding the assessment of cognition in this population, in order to identify areas that can be addressed to improve detection rates in the future. Ethical approval for this study was obtained from the St James Hospital/Tallaght Hospital research ethics committee in December 2011. 


\section{METHODS}

Study site and participants

A 14-item questionnaire was administered to all internal medicine, surgical and ED physicians involved in the acute care of older patients presenting to the ED of a university teaching hospital, with almost $60000 \mathrm{ED}$ visits annually (see box 1 ). Using 'on-call' rosters, and records from the human resources department, all doctors working in the ED, and doctors on medical and surgical services with an ED 'on-call' commitment from 9 January to 5 March 2012, were approached to participate in the study. The usual care pathway for unscheduled attendances in this study setting is outlined in figure 1 .

\section{Questionnaire design}

The questionnaire was developed adopting a Delphi method with inputs from three senior geriatricians and a senior ED physician. As part of this process, this expert panel forwarded specific themes they felt should be addressed by the questionnaire. A prototype of the questionnaire was piloted on a smal sample group from within the department of geriatric medicine, who following feedback and agreement on a final draft, were excluded from further participation.

\section{Questionnaire}

The instrument was self-administered and responses were unaided. Data establishing the specialty, clinical experience and level of exposure to older ED patients was collected. Doctors were then asked to rate the importance of cognitive impairment/dementia relative to other chronic medical conditions (liver, heart, respiratory and renal failure) with regard to its impact on mortality, morbidity and readmission rates, and attribute a score out of 10 for each. Respondents were asked to estimate the prevalence of cognitive impairment in older ED patients, and how frequently they screen for it. They were asked if it was important to screen for cognitive impairment in older ED patients, what the limitations were, and who should perform it. They were asked if they felt an assessment of orientation sufficed as an assessment of cognition, and with regard to their familiarity and use of several established cognitive screening tools in the ED.

\section{Box 1 Details of study setting}

- This study was based in a 625 bed university teaching hospital. Over 60000 patients are reviewed annually in the emergency department (ED), and approximately $12 \%$ are aged 65 or older. The hospital caters for most medical and surgical specialties and is a specialist trauma centre. Emergency and unscheduled care is supported by an acute medical assessment unit, acute stroke unit, coronary care unit and intensive care unit.

- Within the hospital doctor structure in this study setting, trainees usually complete 1 year of medical/surgical internship, followed by 2-3 years as senior house officers within selected specialties, which is when they commence participation in 'on-call' activities in ED. Following this, there is usually a period of at least 5-8 years of speciality training as registrar/specialist registrar within their chosen specialty prior to appointment as consultant.

\section{Data analysis}

As per the stated study objectives, data was collected and collated from completed questionnaires. SPSS 16.0 (SPSS Inc, USA) was used for statistical analysis. Descriptive data of participant's characteristics was presented as frequency and percentage. An estimate of the prevalence of cognitive impairment in older ED patients was presented as mean percentage and SD. An independent $\mathrm{t}$-test was used to examine if completion of a geriatric medicine rotation influenced perceived prevalence of cognitive impairment. An estimate of the percentage of older patients that participants reported screening was also presented as mean and SD. Proportionate comparisons of those who reported a lack of

Patient presents to the emergency department (ED)

Patient is assessed and triaged by an ED nurse specialist

The patient is reviewed by the ED physicians

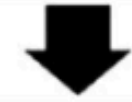

The patient may be directly referred to the Acute Medical Assessment Unit

\section{If necessary, patients are referred onwards for specialist review from medical/surgical specialties}

Figure 1 Pathway of care of unscheduled Emergency Department attendances in the study setting. 
expertise' as a reason for not screening patients more frequently were calculated using the Pearson $\chi^{2}$ test. Similarly, opinions on who should perform cognitive screening were reported using the Pearson $\chi^{2}$ tests. A p value of $<0.05$ was considered statistically significant.

\section{RESULTS}

\section{Details of questionnaire respondents}

A total of 76/97 (78\%) eligible respondents completed the questionnaire. The majority of responders were medical physicians $46 / 76$ (60\%), with surgical and ED physicians accounting for $15 / 76$ (20\%) each (table 1). Consultant physicians and registrars/specialist registrars in geriatric medicine $(n=7)$ were excluded from the sample as they participated in the questionnaire design and piloting phase of the study. All participants were regularly involved in the care of older patients in the ED, with $48 / 76(63 \%)$ treating them on at least a weekly basis, and $25 / 76(33 \%)$ reporting daily contact. $42 / 76(55 \%)$ had spent the equivalent of 3 months or more in a postgraduate geriatrics rotation, while $26 / 61(43 \%)$ of those not currently working in $\mathrm{ED}$ as $\mathrm{ED}$ physicians had previously completed an ED rotation.

\section{Knowledge of cognitive impairment and use of screening instruments in ED}

The mean of respondents' estimates of the prevalence of cognitive impairment in older patients in the ED setting was 44 $\pm 16 \%$ (SD), with $14 / 76$ (18\%) estimating a prevalence of $\leq 20 \%$. More than a quarter of respondents $(21 / 76,28 \%)$ overestimated the prevalence by estimating that $\geq 60 \%$ of older people attending the ED were cognitively impaired. Completion of a training rotation in geriatric medicine did not significantly influence a respondent's perception of the prevalence of cognitive impairment in older ED patients $(44.9 \pm 21 \%$ geriatric rotation, vs $36.5 \pm 18.4 \%$ no geriatric rotation, $t$-test $=1.83$, $\mathrm{p}=0.07$ ).

Over a quarter of respondents $(19 / 76,26 \%)$ felt a simple assessment of orientation to person, place and time, sufficiently assessed cognition. The Mini Mental State Examination (32/76, $42 \%)$, and the Abbreviated Mental Test Score (29/76, 38\%), cognitive tests were the most frequently used screening tools in the ED. Otherwise there was very little use of, or familiarity with, other established brief cognitive screening tools, which are

Table 1 Demographics of questionnaire participants

\begin{tabular}{|c|c|c|c|}
\hline & $\begin{array}{l}\text { ED Physicians } \\
(n=15)\end{array}$ & $\begin{array}{l}\text { Medical } \\
\text { physicians } \\
(n=46)\end{array}$ & $\begin{array}{l}\text { Surgeons } \\
(n=15)\end{array}$ \\
\hline Gender, male, n (\%) & $8(53)$ & $18(39)$ & $11(73)$ \\
\hline Consultant, n (\%) & $3(20)$ & $8(17)$ & $3(20)$ \\
\hline Registrar/Specialist Registrar, n (\%) & $3(20)$ & $20(44)$ & $7(47)$ \\
\hline Senior House Officer, $\mathrm{n}(\%)$ & $9(60)$ & $18(39)$ & $5(33)$ \\
\hline Qualified $\geq 6$ years, $n(\%)$ & $7(47)$ & $19(41)$ & $9(60)$ \\
\hline Qualified $4-6$ years, $n(\%)$ & $2(13)$ & $9(20)$ & $1(6.7)$ \\
\hline Qualified 2-4 years, $n(\%)$ & $1(6.7)$ & $12(26)$ & $3(20)$ \\
\hline Qualified $\leq 2$ years, $n(\%)$ & $5(33)$ & $6(13)$ & $2(13)$ \\
\hline Completed GM Rotation, n (\%)* & $7(47)$ & $30(65)$ & $5(33)$ \\
\hline Completed ED Rotation, $\mathrm{n}(\%)^{*}$ & - & $19(41)$ & $7(47)$ \\
\hline $\begin{array}{l}\text { Older patient review in ED at least } \\
\text { weekly }\end{array}$ & $11(73)$ & a25 (54) & $12(80)$ \\
\hline
\end{tabular}

often considered as more suitable for use in the ED: Six Item Screener $(4 / 76,5 \%)$, Mini-cog $(4 / 76,5 \%)$, Clock Drawing Test $(0 / 76,0 \%)$, Short Blessed Test $(0 / 76,0 \%)$, Ottawa 3DY (1/76, $1 \%)$, Montreal Cognitive Assessment (2/76, 3\%) and Orientation Concentration Memory Test $(2 / 76,3 \%)$.

\section{Perceived skills in the assessment and identification of cognitive impairment}

Respondents routinely screened less than one in four older patients they reviewed for cognitive impairment (mean, 24 $\pm 20 \%$ ); with $33 / 76$ (43\%) reporting they screened less than one in ten. Over a quarter $(22 / 76,29 \%)$ felt they lacked the relevant expertise to perform cognitive screening. Proportionately, respondents who had never completed a rotation in geriatrics were more likely to report a 'lack of expertise' as a limiting factor $(14 / 34(41 \%)$ vs $8 / 42$ (19\%), Pearson $\left.\chi^{2}=4.47, \mathrm{p}=0.034\right)$. Similarly, ED physicians $(8 / 15,53 \%)$ and surgeons $(6 / 15,40 \%)$ were more likely to report lack of expertise as a limiting factor, compared to medical physicians $(8 / 46$ (17\%), Pearson $\chi^{2}=8.22, p=0.016$ ). The number of years qualified did not affect a doctor's own perceptions of expertise. Over half of all respondents $(39 / 76,51 \%)$, cited the lack of availability of a screening instrument as a major limiting factor. Lack of privacy $(47 / 76,62 \%)$, too much noise $(55 / 76,72 \%)$ and time constraints $(54 / 76,71 \%)$, were also reported as significant limiting factors to the performance of cognitive screening.

Attitudes towards cognitive impairment in older ED patients Despite the low numbers of older patients routinely screened, the majority of doctors $59 / 76(78 \%)$ felt cognitive screening in this population group was important in the ED setting. There was a difference of opinion about who should perform cognitive screening in older ED patients, with 11/15 (73\%) of doctors from surgical departments considering that ED physicians should perform screening, 10/15 (66\%) of ED physicians considering that the admitting medical physician or surgeon should perform screening, and 21/46 (46\%) of medical physicians considering that the ED physician or nurse should perform screening (Pearson $\chi^{2}=19.7, p=0.012$ ).

When asked to rate the importance of cognitive impairment/ dementia in terms of its relative impact on patient mortality, morbidity and readmission risk, compared to other chronic diseases, it was ranked fourth after lung, heart and liver disease, but placed ahead of chronic kidney disease (table 2).

\section{DISCUSSION}

This is the first study to report on factors that may contribute to the inadequate detection of cognitive impairment in older ED patients, by doctors working in the ED. This survey highlights how potential deficiencies in the knowledge, skills and attitudes

Table 2 Perceived importance of chronic conditions in terms of impact on patient mortality, morbidity and readmission risk

\begin{tabular}{lll}
\hline Rank in order of importance & Chronic condition & Mean (SD)* \\
\hline 1 & Respiratory disease & $7.4(1.5)$ \\
2 & Heart disease & $7.1(1.7)$ \\
3 & Liver disease & $6.9(1.9)$ \\
4 & Cognitive impairment/dementia & $6.7(2.1)$ \\
5 & Kidney disease & $6.3(1.6)$ \\
\hline *Mean score out of ten, where higher score indicates greater importance
\end{tabular}


of doctors may be contributing to this inadequate detection. Respondents in this survey were experienced doctors, regularly involved in the care of older patients in the ED setting. While the overall estimation of the prevalence of cognitive impairment in older ED patients was reasonably accurate, almost half of the doctors surveyed significantly overestimated, or underestimated it. There was little familiarity with, or use of brief cognitive screening instruments in ED. Doctors reported they screened very few older patients, and a lack of expertise appeared to be a prominent factor in this. The lack of a standard screening instrument, ED environmental factors such as a lack of privacy and noise, and general time constraints were also commonly reported as significant issues limiting a doctor's performance in this regard. The vast majority of doctors surveyed felt it was necessary and important to screen older patients in ED for evidence of cognitive impairment, but there was little consensus as to who should do this.

Despite older patients representing an increasing proportion of the workload in the ED, almost half of the respondents had never completed a geriatrics training rotation, and this appeared to influence their own perception of competency for identifying, and treating cognitively impaired older patients. This was also evident in the relatively low prioritisation accorded to cognitive impairment/dementia in terms of other organ failures with regards to impact on morbidity, mortality and rehospitalisation, even though dementia is considered the leading cause of disability and dependency in older persons worldwide. ${ }^{20}$ While the training and education of ED staff in gerontological principles should be a core competency as recommended for all healthcare workers, ${ }^{21}$ and has been shown to yield care dividends, ${ }^{22}$ we would consider that there may be a particular added benefit from specialist geriatrician input in the acute care setting. Their role would be to deliver immediate targeted care for older patients, appropriate forward referral for assessment, and to take leadership of developing targeted education programmes for healthcare staff in these areas. ${ }^{23}$

Within the ED literature there has been a welcome increase in attention towards the recognition and diagnosis of acute delirium in previously cognitively well individuals, but a relative lack of attention to the syndrome of pre-existing dementia. Geriatricians frequently review patients who have acute delirium superimposed on a background of dementia which has not been detected or evaluated prior to the current admission. Similarly, many patients with pre-existing, but undiagnosed dementia are often treated in ED for an epiphenomenon of their dementia (eg, falls), but they are discharged home without formal review and diagnosis of their dementia. When formal cognitive screening is performed on older patients in the ED, over $80 \%$ of those who screen positive have no prior history of dementia. ${ }^{12}$ Doctors in this survey reported that the absence of a familiar standardised screening tool limited their ability to screen more patients. This is despite the existence of several validated brief screening tools for the ED setting. ${ }^{24}$ Given that there is no consensus in the literature in favour of one particular tool, we would recommend that it is important that ED units adopt a single screening tool, incorporate it into their practice and ensure adequate training of staff in its administration and interpretation. Recent Department of Health guidelines in the UK have recommended the use of the Abbreviated Mental Test- 4 score as a quick screening tool for cognition in all older ED patients (http://www.dh.gov.uk/publications). Clarification of a diagnosis of dementia, delirium or delirium superimposed on a background of dementia can only be established with the careful collection of a collateral history from a person who knows the patient well, which with the completion of a cognitive screening test should be an integral part of the routine clinical work-up for all older patients in ED.

Environmental factors and time constraints were cited as limitations to assessing cognition in the ED setting. It is clear that the physical infrastructure of ED is not catering to the needs of this growing number of older patients, or the doctors who are treating them. There is a necessity for more private consultation areas to assess cognition and to obtain a clear collateral history. Environmental modification of ED requires systematic planning and should to cater to the complex needs of patients with cognitive impairment. Good design with effective use of lighting, colour contrast, noise-limiting measures and clear orientation cues can significantly affect someone's ability to function and understand within perhaps the most challenging hospital environment. There was no clear consensus as to who is best placed to screen patients for cognitive deficits, with each speciality feeling it was another's responsibility, with the obvious default that in most instances screening does not occur. Given that the initial patient assessment is performed in most instances by ED doctors, and they have most contact with both admitted and discharged patients, we would suggest that targeted training of this group is likely to have greatest effect on detection rates.

This study addressed several of the issues limiting the effective screening of older patients in ED. Overall there was a reasonably good response rate to the questionnaire, enabling a better understanding of these issues from the perspectives of medical, surgical and ED doctors. The generalisability of these results may be limited due to this study being conducted at a single academic urban hospital, although many respondents would have worked in several other hospitals and jurisdictions during their training. Although the response rate to this survey was reasonable, no response was obtained from 21/97 (22\%) eligible participants. This may have biased our results, although it is possible this group may have been less likely to test for cognitive impairment or consider it a priority. The results and conclusions in this report are based on what doctors reported they do; in reality their performance may be substantially worse than this.

\section{Main messages}

There is a high prevalence of cognitive impairment and delirium in older emergency department (ED) patients, and repeated studies have reported inadequate detection and documentation by doctors.

- Despite many doctors acknowledging the importance of cognitive screening in older ED patients, on average they screen less than one-in-four patients

- Important limitations to effective cognitive screening in ED included environmental factors (lack of privacy and noise), perceived lack of expertise, time constraints and the absence of a standardised brief screening tool.

- Absence of structured gerontological training appeared to influence doctor's perceptions of competency for identifying cognitive impairment.

- Development of a gerontologically attuned ED environment, incorporation of routine cognitive screening into the 'work up' of older patients and clear pathways for follow-up care would likely enhance ED care of this vulnerable group. 


\section{Current research questions}

Which brief cognitive screening instrument is best suited for screening older patients in emergency department (ED)?

- How would implementation of a structured education programme targeted at physicians and nurses caring for older patients in acute settings influence detection, diagnosis and documentation rates in older ED patients?

- How many patients identified with cognitive impairment in the ED will be diagnosed with dementia when followed up and assessed at a later date?

\section{Key references}

- Aminzadeh F, Dalziel WB. Older adults in the emergency department: a systematic review of patterns of use, adverse outcomes, and effectiveness of interventions. Ann Emerg Med 2002;39:238-47.

- Samaras N, Chevalley T, Samaras D, et al. Older patients in the emergency department: a review. Ann Emerg Med 2010;56:261-9.

- Hirschman KB, Paik HH, Pines JM, et al. Cognitive impairment among older adults in the emergency department. West J Emerg Med 2011;12:56-62.

- Han JH, Wilson A, Ely EW. Delirium in the older emergency department patient: a quiet epidemic. Emerg Med Clin North Am 2010;28:611-31.

- Hustey FM, Meldon S, Palmer R. Prevalence and documentation of impaired mental status in elderly emergency department patients. Acad Emerg Med 2000;7:1166.

The evidence supports targeted screening of all older ED patients for delirium, cognitive impairment and dementia, but despite repeated and consistent results from studies indicating high prevalence and low detection rates the practice has not developed in most centres. The incorporation of a brief cognitive screening assessment, combined with a focus on the attainment of a clear collateral history should be considered part of the routine examination of all older ED patients. In addition, clinical pathways for further assessment, management and appropriate referral of those found to have dementia and/or delirium need to be clarified and implemented, including protocols for the prevention of emergent delirium in those at risk. ${ }^{25}$ Future studies need to assess the effectiveness of specific training programmes for healthcare staff working in ED, and specifically measure outcomes on the detection and management of cognitive impairment in older patients.

Acknowledgements The authors gratefully acknowiedge the contribution of Dr Anna Moore and Dr Aoife 0 Carroll for their assistance in distributing the questionnaire.
Contributors SK: Questionnaire design, data analysis and interpretation, manuscript preparation and editing, DM, TC and RC: Questionnaire design, questionnaire distribution and manuscript preparation, MR and DO: Questionnaire design, data analysis and interpretation, manuscript preparation and editing. Competing interests None.

Ethics approval Tallaght and St James Hospital ethics committee.

Provenance and peer review Not commissioned; externally peer reviewed

\section{REFERENCES}

1 Roberts DC, MCKay MP, Shaffer A. Increasing rates of emergency department visits for elderly patients in the United States, 1993 to 2003. Ann Emerg Med 2008:51:769-74.

2 Hu SC, Yen D, Yu YC, et al. Elderly use of the ED in an Asian metropolis. Am $\int$ Emerg Med 1999;17:95-9.

3 Pitts SR, Niska RW, Xu J, et al. National hospital ambulatory medical care survey: 2006 emergency department summary. Natl Health Stat Report 2008;6:1-38.

4 Aminzadeh F, Dalziel WB. Older adults in the emergency department: a systematic review of patterns of use, adverse outcomes, and effectiveness of interventions. Anm Emerg Med 2002:39:238-47.

5 Samaras N, Chevalley T, Samaras D, et al. Older patients in the emergency department: a review. Ann Emerg Med 2010;56:261-9.

6 Grief $\mathrm{CL}$. Patterns of ED use and perceptions of the elderly regarding their emergency care: a synthesis of recent research. J Emerg Nurs 2003;29:122-6.

7 Hwang U, Morrison RS. The geriatric emergency department. J Am Gerlatr Soc 2007;55:1873-6.

8 Gerson LW, Counsell SR, Fontanarosa PB, et al. Case finding for cognitive impairment in elderly emergency department patients. Ann Emerg Med 1994;23:813-17.

9 Martin M, Hin PY, O'Neill D. Acute medical take or subacute-on-chronic medical take? If Med / 2004:97:212-14.

10 Hirschman KB, Paik HH, Pines JM, et al. Cognitive impairment among older adults in the emergency department. West J Emerg Med 2011:12:56-62.

11 Han JH, Wilson A, Ely EW. Delirium in the older emergency department patient a quiet epidemic. Emerg Med Clin North Am 2010;28:611-31.

12 Hustey FM, Meldon SW. The prevalence and documentation of impaired mental status in elderly emergency department patients. Ann Emerg Med 2002;39:248-53.

13 Hustey FM, Meldon S, Palmer R. Prevalence and documentation of impaired mental status in elderly emergency department patients. Acad Emerg Med 2000;7:1166.

14 Hustey FM, Meldon SW, Smith MD, et al. The effect of mental status screening on the care of elderly emergency department patients. Ann Emerg Med 2003:41:678-84.

15 Wilber ST. Aitered mental status in older emergency department patients. Emerg Med Clin North Am 2006:24:299-316, vi.

16 Seltzer B, Zolnouni P, Nunez M, et al. Efficacy of donepezil in early-stage Alzheimer disease: a randomized placebo-controlled trial. Arch Neurol 2004;61:1852-6.

17 Baneriee S, Wittenberg R. Clinical and cost effectiveness of services for early diagnosis and intervention in dementia. Int J Geriatr Psychiatry 2009;24:748-54.

18 lliffe S. Wilcock J, Haworth D. Obstacles to shared care for patients with dementia: a qualitative study. Fam Pract 2006:23:353-62.

19 Woodford HJ, George J. Cognitive assessment in the elderly: a review of clinica methods. QMM 2007;100:469-84.

20 Dementia: A public health priority. World Health Organization and Alzheimer's disease international report, April, 2012 (http:Awww.who.int/en.org).

21 Kuwabara $Y$, Ichiya $Y$, Sasaki $M$, et al. Cerebral blood flow and vascular response to hypercapnia in hypertensive patients with leukoaraiosis. Ann NuCl Med 1996;10:293-8.

22 Brymer $C$, Cavanagh $P$, Denomy E, et al. The effect of a geriatric education program on emergency nurses. J Emerg Nurs 2001;27:27-32.

23 Miller DK, Lewis LM, Nork MJ, et al. Controlled trial of a geriatric case-finding and liaison service in an emergency department. J Am Geriatr Soc 1996:44:513-20.

24 Carpenter CR, Bassett ER, Fischer GM, et al. Four sensitive screening tools to detec cognitive dysfunction in geriatric emergency department patients: brief Alzheimer's Screen, Short Blessed Test, Ottawa 3DY, and the caregiver-completed AD8. Acad Emerg Med 2011;18:374-84.

25 Young J, Inouve SK. Delirium in older people. BMJ 2007;334:842-6. 